\title{
Position-assisted fast handover schemes for LTE-advanced network under high mobility scenarios
}

\author{
Ming FEI*, Pingzhi FAN \\ Institute of Mobile Communications, Southwest Jiaotong University, Chengdu 610031, China
}

\begin{abstract}
Two position-assisted fast handover schemes, scheme $A$ and scheme $B$, for LTE-A system under very high mobility scenarios, are proposed, together with their performance evaluation. Scheme $A$ is designed to reduce handover delay by making handover preparation before handover starts. Scheme $B$ aims at reducing unnecessary handovers and improving handover success rate, by calculating the geographically best target handover cell, which makes it easier for mobile terminals to access the target cell. A system level simulation is conducted to evaluate the performance of these two schemes. It is shown that, scheme $A$ could reduce inter-site handover delay by about $50 \mathrm{~ms}$, while scheme $B$ could cut down nearly $50 \%$ of all handovers when time-to-trigger (TTT) is 0 ms. Besides, as TTT gets larger, Scheme $B$ has much better success rate.
\end{abstract}

Key words: LTE-A; handover; very high mobility; handover delay; handover frequency; handover success rate

C 2012 JMT. All rights reserved.

\section{Introduction}

3 GPP has finalized the standardization of the long time evolution (LTE) system within its Release 8 and the LTE-advanced system within Release 10. The requirements of LTE include improved capacity, higher throughput, increased spectrum efficiency, lower latency, and better coverage [1-2].

In LTE system, orthogonal frequency division multiplexing (OFDM) and single carrier frequency domain multiple access (SC-FDMA) are used in downlink and uplink respectively, as radio access schemes [3]. Since the soft handover used in wideband CDMA (WCDMA) system is not applicable here in 3GPP LTE, the hard handover, which causes interruption in the user plane, becomes the sole option. As the radio control functionality in radio access network (RAN) has been distributed to the base stations (eNB) [3], the latency could be significantly reduced in LTE system. Because the radio resource control (RRC) has to be implemented in a distributed way without the assistance of a central control entity, the handover procedure has to be designed according to the distributed nature of LTE.

Received Oct. 21, 2012; revision accepted Nov. 15, 2012

*Corresponding author. E-mail: feimingyy@hotmail.com (M. FEI)

(C) 2012 JMT. All rights reserved

doi: 10.3969/j.issn.2095-087X.2012.04.012
In scenarios with very high mobility, where users travel at very high speeds (e.g. $350 \mathrm{~km} / \mathrm{h}$ ), the handover performance of LTE system is affected by several special problems. First of all, because of the very high user traveling speed, the overlapping area between adjacent cells could be passed in a really short time. In order to maintain the radio link with eNBs, users have to finish the handover procedure as fast as possible, meaning the handover delay should be very small. Secondly, the period of time during which the user stayed in each cell is shorter compared to the scenarios with lower speeds. This requires more handovers, and increases resources occupied by handover procedures. Thirdly, in fading channels with very high mobility, the time variation of the channel over an OFDM symbol period causes a loss of subchannel orthogonality and inter-channel interference (ICI), makes it more difficult to transmit signaling messages, and leads to more radio link failure (RLF) during handover procedure.

On the other hand, LTE-A has standardized three kinds of position technology [4]: enhanced cell ID (Ecell ID), observed time difference of arrival (OTDOA), and assisted global navigation satellite system (AGNSS). The development of these positioning technologies makes it possible for LTE-A system to obtain position information with high accuracy, which could help us improve the handover performance of LTE-A system [5]. 


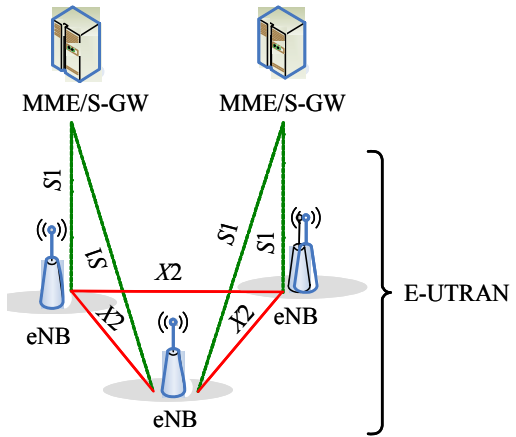

Fig. 1 Overall architecture of LTE-A [3]

Some researchers have investigated the handover problem. In Ref. [6], the impacts of triggering setting (hysteresis/TTT) on handover performance in LTE system have been investigated for different scenarios of low, medium and high system loads. Kitagawa and Komine [7] proposed a self-optimization algorithm for handover (HO) parameters which was robust against the change in user (UE) mobility. A seamless handover scheme for high speed rail was proposed in Ref. [8], in which two antennas were mounted at the front and rear of a train respectively, and one of them executed handover while the other was still communicating with BS. Simulation and theoretical results showed that this scheme could efficiently support seamless handover with low overhead. Luo et al. [9] proposed a scheme that adjusts TTT and entering and leaving condition of trigger handover at different positions. Simulation results showed that proposed scheme decreased outage probability and improved handover success probability. Wang et al. [10] proposed an adaptive handover trigger scheme to guarantee a high handover triggered probability by configuring overlapped area and measurement report period adaptively according to the speed. A soft handover algorithm for TD-LTE system was proposed in Ref. [11] for high speed railway scenario. Simulation results showed that the proposed algorithm kept a stable signal quality and decreased the number of handover at the same time.

This paper proposes two position-assisted fast handover schemes that can be used to reduce handover delay, handover frequency and handover failure rate. These schemes, taking into account of the user locations during the handover procedure, can be used to predict target handover cells and change handover parameters, and thus improve the handover performance. In order to verify the effectiveness of the proposed schemes, system level simulation is conducted, and simulation results show that proposed schemes can greatly improve handover performance.

\section{LTE-advanced handover scheme}

In LTE system, handover procedure can be illustrated as Fig. 2. Handover starts with the measurement report (MR) sent by user equipment (UE) to serving eNB. Based on the received measurement reports, involved eNB chooses the target eNB and makes handover preparation.

The handover preparation includes signaling between serving eNB and target eNB, and admission control of UE in target eNB. If target eNB permits the access of UE and the handover preparation succeeds, handover command will be sent to UE by serving eNB, and the connection between UE and serving eNB will be released. Upon successful synchronization with the target eNB, UE connects to this new eNB and responds with handover confirm message. Then handover procedure is successfully finished.

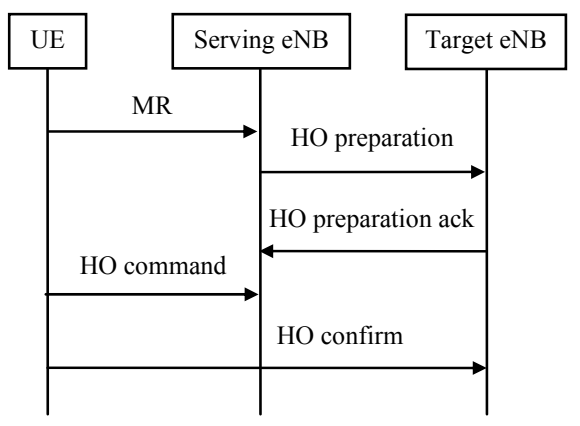

Fig. 2 Handover procedure

As mentioned above, the handover procedure is triggered at UE based on the triggering algorithms and parameters defined by the network.

UE periodically performs downlink radio channel measurement based on the reference symbols (RS); namely UE can measure the reference symbol received power (RSRP) and the reference symbol received quality (RSRQ). Both RSRP and RSRQ can be used in the handover triggering algorithms.

Several events have been defined in LTE to describe handover triggering algorithms in different scenarios. In case the intra-frequency handover is performed based on RSRP values, a measurement report is transmitted when the following criterion is met:

RSRPneighbor and RSRPserving are the neighbor and serving cell RSRP measurements. Handover margin (HOM) and time-to-trigger (TTT) are the parameter sets defined by the network. Handover is triggered when the RSRP value from the adjacent cell is higher than the one from serving cell by a number of $\mathrm{dBs}$ equal to HOM, and this condition has to be satisfied for a period equal to TTT, as shown in Fig. 3. 


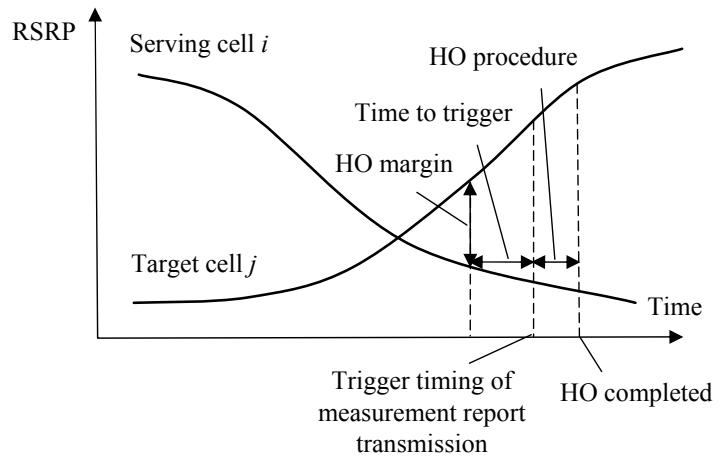

Fig. 3 Handover decision algorithm

Before being used in handover triggering algorithm, the RSRP values measured in a measurement period are usually averaged by L1 filter, and then the output of L1 filter will be processed by L3 filter, as Eq. (1) shows:

$$
R S R P_{n}=\left[1-\left(\frac{1}{2}\right)^{4 / K}\right] \times R S R P_{n-1}+\left(\frac{1}{2}\right)^{4 / K} \times q_{n} .
$$

where $R S R P_{n}$ and $R S R P_{n-1}$ are updated and previous RSRP values; $q_{n}$ is the current measure value produced by L1 filter; $K$ is L3 filter coefficient.

The appropriate setting of $\mathrm{HO}$ triggers is of significant importance to the $\mathrm{HO}$ procedure, since it decides when the handover is triggered and the radio channel state at that instant. The optimal setting depends on UE speed, radio network deployment, propagation conditions, and the system load.

In the scenarios where UEs move very fast, several special problems should be taken into consideration. First of all, the radio network deployment changed. In order to avoid frequent handovers caused by the high speed, cells become larger and the signal conditions at the cell edge degrades, causing more handover failure. Secondly, the optimal settings of HOM and TTT are different in low and high mobility scenarios. Thirdly, the serious Doppler effects in high speed scenarios will cause ICI in OFDM system, the signaling messages during the handover procedure may not be successfully received. In order to improve the handover performance in high mobility scenarios, two position assisted handover schemes are proposed in the next section.

\section{Position-assisted handover schemes}

In this section, two position-assisted fast handover schemes are proposed for high mobility scenarios, one of which aims at reducing handover delay, and the other focuses on reducing handover frequency and improving handover success rate. Both of the schemes take advantage of UE position information to help improve handover performance.

\subsection{Scheme A}

In inter-site handover procedure, after receiving measurement report, the handover preparation has to be conducted between serving eNB and target eNB, causing significant delay due to the signaling through $\mathrm{X} 2$ interface. Scheme $A$ tries to reduce this part of delay, by making use of UE position information. Fig. 4 shows the handover procedure of scheme $A$.

In scheme $A$, suppose that UE positions are available and the locations of adjacent eNB are known. When UE detects that the distance between UE and its serving cell is larger than a predefined value, a prediction request message is sent to the network. Then the potential target handover cell is predicted by serving eNB, and handover preparation can be conducted before measurement report is sent. When the handover starts, if the predicted target handover cell is the same as the one that MR suggested, the delay caused by handover preparation can be saved; the overall handover delay is reduced to $\mathrm{Td}$, as shown in Fig. 4. Assume that each X2 transfer and eNB processing of a message requires $10 \mathrm{~ms}$, the entire intereNB handover preparation costs $60 \mathrm{~ms}$, which is the time saved in a success prediction action.

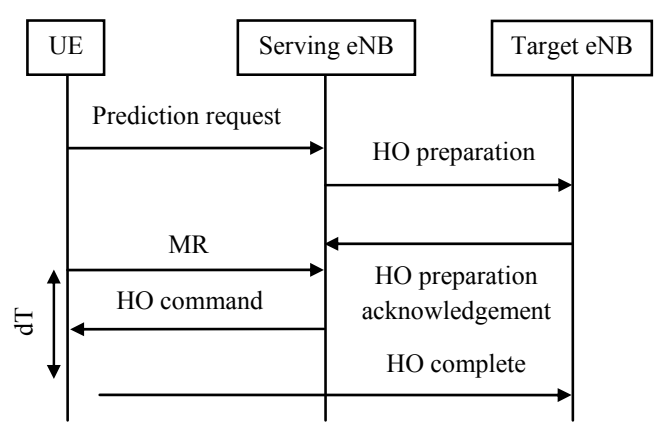

Fig. 4 HO procedure of scheme $A$

However, if the predicted target handover cell is wrong, the handover preparation still has to be carried out after the serving eNB receiving MR, and then the handover delay will not be lower. Hence, the target handover cell prediction algorithm is very important.

Suppose that the location of UE is denoted by $\left(X_{u}, Y_{u}\right)$, the locations of serving eNB and adjacent eNB are denoted by $\left(X_{0}, Y_{0}\right)$ and $\left(X_{i}, Y_{i}\right)$, and $i$ takes the value of 1 to 6 ( 6 adjacent sites are considered). Then the predicted target handover cell can be calculated as:

$$
\begin{aligned}
& \text { PredictedTargetCellID }=\arg \min _{i} \times \\
& \qquad\left\{\text { dis }_{i} \times \cos \left[\left|\arg \tan \left(\frac{Y_{i}-Y u}{X_{i}-X u}\right)-\arg \tan \left(\frac{Y_{i}-Y_{0}}{X_{i}-X_{0}}\right)\right|\right]\right\}
\end{aligned}
$$

and 


$$
d i s_{i}=\sqrt{\left(X u-X_{i}\right)^{2}+\left(Y u-Y_{i}\right)^{2}} .
$$

In fact, for each adjacent eNB $i$, the algorithm calculates the length of $N_{1} H_{1}$ and $N_{2} H_{2}$, as shown in Fig. 5; then the eNB with the shortest distance between point $N_{i}$ and point $H_{i}$, becomes the target handover eNB.

Scheme $A$ is dedicated to reducing the overall handover delay. The handover frequency and success rate problems are discussed in Scheme $B$.

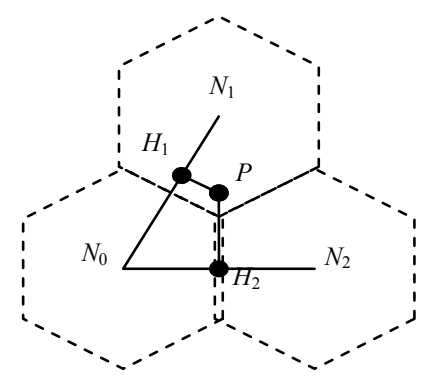

Fig. 5 Target cell prediction algorithm

\subsection{Scheme B}

In handover scenario, a lot of handovers are triggered because of the existing shadow fading and fast fading channel, even though many of them are unnecessary. Hence the handover frequency is increased and the success rate declines. Scheme $B$ is designed to avoid some of these unnecessary handovers and improve handover success rate by the use of UE position information.

With the UE location (serving/adjacent eNB location), and the antenna pattern of serving/adjacent cells, we can approximately calculate the large scale fading. According to the large scale fading of each cell, a geographically best target handover cell with the smallest large scale fading value could be found for UE.

For example, a distance-dependent path loss formula for macro-cell system is defined [11]:

$$
\text { Pathloss }=128.1+37.6 \times \log R, R \text { in } \mathrm{km}
$$

and an antenna pattern of 3-sector sites that is known to eNB,

$$
A_{V}(\theta)=-\min \left[12\left(\frac{\theta}{\theta_{3 \mathrm{~dB}}}\right)^{2}, S L A_{v}\right],
$$

both $R$ and $\theta$ can be calculated by the location of UE, then we can get the approximate large scale fading between UE and each cell,

$$
\text { Fading }_{\text {large-scale }}=\text { Pathloss }-A_{v}(\theta) \text {. }
$$

Finally the predicted best target handover cell is chosen by Eq. (5).

In order to avoid the unnecessary handover caused by shadow and fast fading, a positive offset parameter is added to the RSRP of this predicted target handover cell, making it easy for UE to connect to this cell, as shown in Fig. 6.

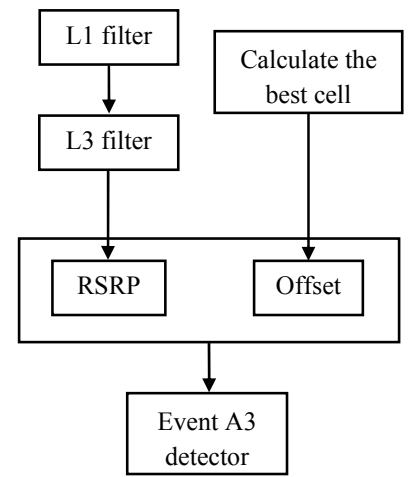

Fig. 6 RSRP calculation of scheme $B$

Thus the RSRP can be calculated as:

$$
R S R P_{n}=\left[1-\left(\frac{1}{2}\right)^{4 / K}\right] \times R S R P_{n-1}+\left(\frac{1}{2}\right)^{4 / K} \times q_{n}+\text { Offset. }
$$

where Offset could be set as 0 or some predefined positive value, according to whether this cell is the predicted best target handover cell.

Scheme $B$ could counterbalance some of the effect of shadow fading and fast fading, thus reduce handover frequency and handover failures.

\section{Simulation results and discussions}

A system level simulation is performed to evaluate the LTE handover schemes and the proposed handover schemes. The handover simulation model is based on Ref. [12].

The radio network used in the simulator consists of 19 sites and 57 cells. Each site serves 3 cells and host 1 eNB. Table 1 shows the detailed simulation parameters and their values.

Path loss, shadow fading, and fast fading are included in the simulation. Path loss is modeled according to a distance based formula with a center frequency of $2 \mathrm{GHz}$. The shadow fading is modeled as log-normal distributed with mean value of $0 \mathrm{~dB}$ and a standard deviation of $8 \mathrm{~dB}$. The spatial de-correlation distance is set to $50 \mathrm{~m}$, and inter-site correlation parameter is set to 0.5 .

In order to evaluate the handover performance in high mobility scenarios, the speed of UE is set as $350 \mathrm{~km} / \mathrm{h}$ during all the simulations performed.

When simulation starts, UE is uniformly generated at the center of the map, moving with the constant 
speed set by the system and the direction randomly generated at the beginning of the simulation. Simulation ends with UE moving to the area outside the map, as shown in Fig. 7.

The performance of handover procedure is measured in terms of $\mathrm{HO}$ success rate, $\mathrm{HO}$ delay, and $\mathrm{HO}$ frequency.

(1) HO success rate: A handover is considered as failure when the transmission of one RRC handoverinvolved message fails. Radio link failure detected during a handover procedure is also considered as $\mathrm{HO}$ failure.

Table 1 Simulation parameters

\begin{tabular}{ll}
\hline Parameters & Values \\
\hline Cell layout & 19 sites, 57 cells \\
ISD & 3000 \\
Path loss & $128.1+37.6 \log R, R$ in km \\
Shadowing standard deviation & $8 \mathrm{~dB}$ \\
Correlation distance of shadow & $50 \mathrm{~m}$ \\
Fast fading & 1 -path Rayleigh fading \\
& $A_{V}(\theta)=$ \\
Antenna pattern & $-\mathrm{min}\left[12\left(\frac{\theta}{\theta_{3 \mathrm{~dB}}}\right)^{2}, S L A_{v}\right.$ \\
& $\theta_{3 \mathrm{~dB}}=70, S L A_{V}=20 \mathrm{~dB}$ \\
Carrier frequency & $2 \mathrm{GHz}$ \\
UE Speed & $350 \mathrm{~km} / \mathrm{h}$ \\
UE direction & {$[0,2 \pi]$} \\
TTT of interest & $0,80,160,320 \mathrm{~ms}$ \\
HOM of interest & $0,1,2,3,4,5 \mathrm{~dB}$ \\
L3 filter coefficient & $k=4$ \\
BS TX power & $46 \mathrm{dBm}$ \\
UE TX power & $24 \mathrm{dBm}$ \\
Traffic load & 0.2 \\
\hline & \\
\hline &
\end{tabular}

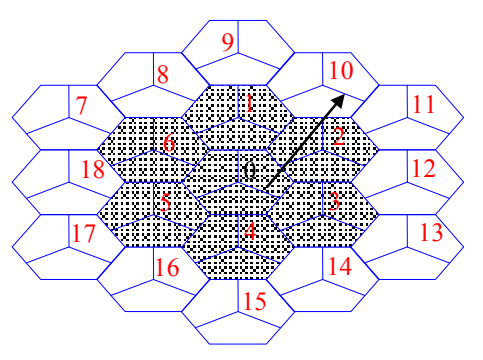

Fig. 7 UE moving pattern
(2) HO delay: The overall delay of a handover is defined as the duration between UE sending meassurement report and target eNB receiving the $\mathrm{HO}$ confirm message. Hence, HO delay is included.

(3) HO frequency: handover frequency is counted as the number of handover initiated in simulation.

Now, we evaluate the performance of the proposed position-assisted fast handover scheme $A$ and scheme $B$, and compare them with LTE-A handover scheme. In all simulations presented here, we assume that 200 simulations are taken for each set of scheme and parameter setting.

The simulation results of $\mathrm{HO}$ success rate, $\mathrm{HO}$ delay, and $\mathrm{HO}$ frequency are presented in Figs. 8-10, some of which will be compared with those in Ref. [13]. For all the simulation results shown below, UE speed is $350 \mathrm{~km} / \mathrm{h}, \mathrm{HOM}$ is $3 \mathrm{~dB}$, and offset is $0 \mathrm{~dB}$ or $3 \mathrm{~dB}$.

From the simulation results, as shown in Figs. 8-9 that scheme $A$ could reduce HO delay by about $50 \mathrm{~ms}$ compared to LTE-A handover scheme, and HO success rate is almost the same.

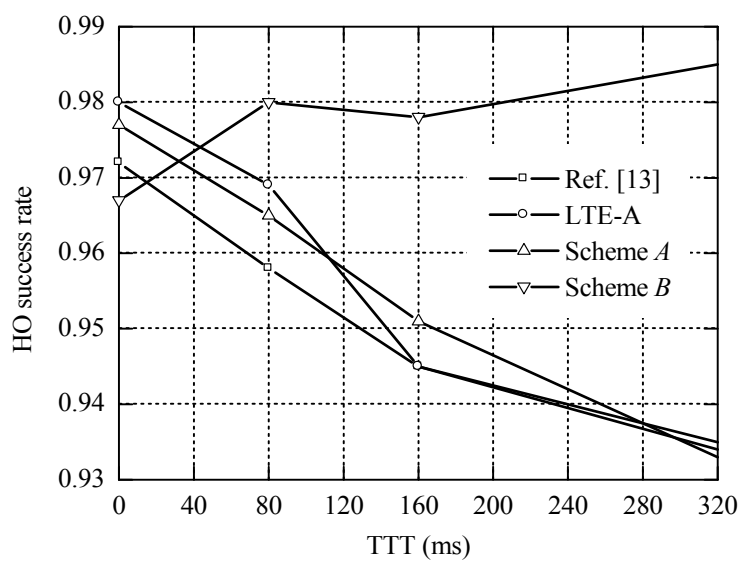

Fig. $8 \mathrm{HO}$ success rate of 3 handover schemes

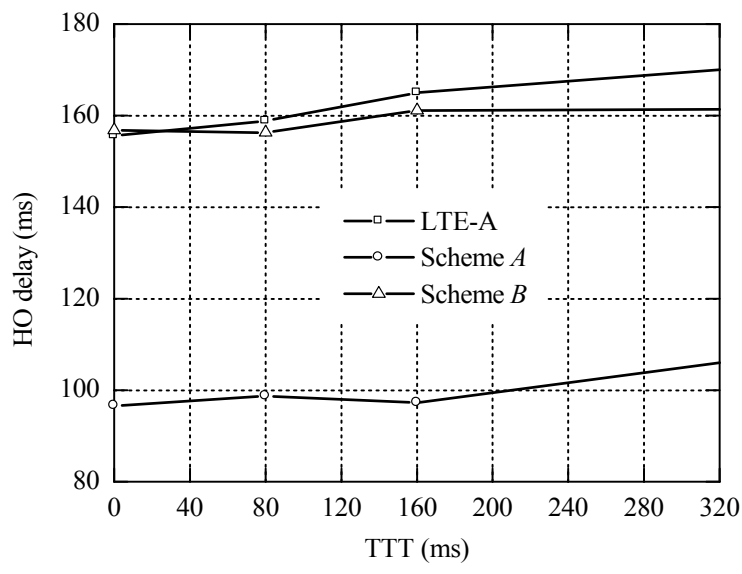

Fig. 9 Inter-site HO delay of 3 handover schemes 


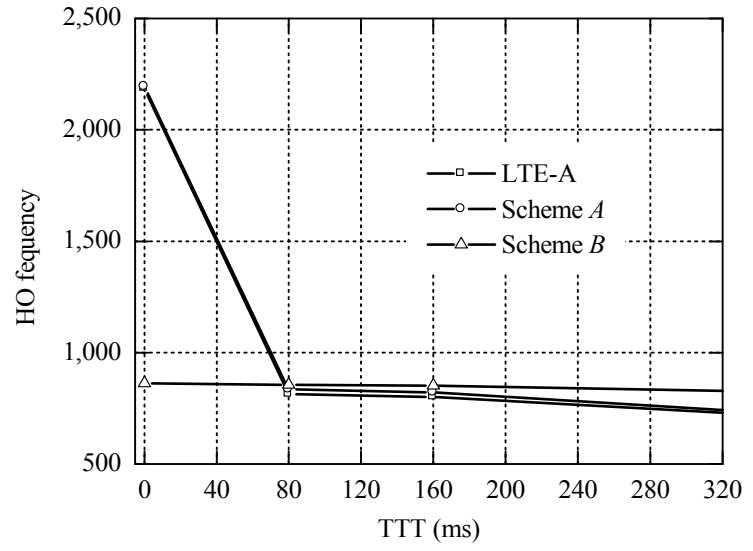

Fig. $10 \mathrm{HO}$ frequency of 3 handover schemes

From Figs. 8 and 10, Scheme $B$ could significantly reduce handover frequency when TTT is small, but the $\mathrm{HO}$ success rate is slightly lower than that of LTE-A handover schemes, because scheme $B$ makes it more difficult for UE to handover to the cell with best signal quality if there is a deep fading area. However, from Fig. 8 , when TTT is larger, scheme $B$ could significantly improve $\mathrm{HO}$ success rate by making it easier and faster for UE to access to the best cell.

\section{Conclusions}

In this paper, two position-assisted fast handover schemes have been studied to reduce the handover delay, handover frequency, and to improve success rate of LTE-A systems under high mobility scenario. Both schemes take into account the UE position information. Scheme $A$ uses UE position to predict the potential target handover cell and makes handover preparation before handover procedure starts. Scheme $B$ calculates the geographically best target handover cell by using the UE position information and adding a positive offset parameter to its RSRP, resulting in significantly reduced handovers when TTT is small and in improved success rate when TTT is larger.

\section{Acknowledgments}

This work was supported by the National Natural Science Foundation of China (No. 61032002), the National Basic Research Program of China (973 Program No. 2012CB316100), and the 111 project (No. 111-2-14). The authors would like to thank Miss Ziyue Liu for her insightful comments and discussions.

\section{References}

[1] 3GPP TS 36.133, 3rd Generation Partnership Project; technical specification group radio access networks; requirements for support of radio source management (Release 8).

[2] [IMT.EVAL] Guidelines for evaluation of radio interface technologies for IMT-Advanced.

[3] 3GPP TS 36.300, V8.5.0, 3rd Generation Partnership Project; technical specification group radio access Network; evolved universal terrestrial radio access (EUTRA) and evolved universal terrestrial radio access Network (E-UTRAN); overall description; Stage 2 (Release 8), 2008.

[4] 3GPP TS 36.355, V9.2.1, 3rd Generation Partnership Project; technical specification group radio access network; evolved universal terrestrial radio access (EUTRA) and evolved universal terrestrial radio access network (E-UTRAN); LTE Positioning Protocol (Release 9), 2010.

[5] 3GPP TR 25.814, V7.1.0, 3rd Generation Partnership Project; technical specification group radio access Network; Physical layer aspects for evolved Universal Terrestrial Radio Access (UTRA) (Release 7), 2006.

[6] C.C. Lin, Optimization of handover algorithm in 3GPP LTE, In: 4th International Conference on Modeling, Simulation and Applied Optimization, Kuala Lumpur, 2011: 1-5.

[7] K. Kitagawa, T. Komine, A handover optimization algorithm with mobility robustness for LTE systems, In: International Symposium on Personal, Indoor and Mobile Radio Communications, Toronto, 2011: 1647-1651

[8] L. Tian, Y.Q. Zhou, J. Li, et al., A novel handover scheme for seamless wireless connectivity in high-speed rail, In: IEEE 7th International Conference on Wireless and Mobile Computing, Networking and Communications, Wuhan, 2011: 230-236.

[9] W.T. Luo, X.M. Fang, M. Cheng, et al., An optimized handover trigger scheme in LTE systems for high-speed railway, In: The 5th International Workshop on Signal Design and its Application in Communications, Guilin, 2012: 193-196.

[10] J. Li, L. Tian, Y.Q. Zhou, et al., An adaptive handover trigger scheme for wireless communications on high speed rail, In: IEEE International Conference on Communications, Ottawa, 2012: 5185-5189.

[11] Q.W Wang, G.L. Ren, J. Tu, A soft handover algorithm for TD-LTE system in high-speed railway scenario, In: IEEE International Conference on Signal Processing, Communications and Computing, Xi'an, 2011: 1-4.

[12] R2-092433, NTT DOCOMO, Evaluation model for Rel8 mobility performance.

[13] R2-093273, NTT DOCOMO, Evaluation of Rel-8 LTE mobility performance. 